\title{
Locoregional and systemic therapy for hepatocellular carcinoma
}

\author{
Olumide B. Gbolahan ${ }^{1}$, Michael A. Schacht ${ }^{2}$, Eric W. Beckley ${ }^{2}$, Thomas P. LaRoche ${ }^{2}$, Bert H. O'Neil ${ }^{1}$, \\ Maximilian Pyko
}

${ }^{1}$ Division of Hematology Oncology, ${ }^{2}$ Department of Interventional Radiology, Indiana University School of Medicine, Indianapolis, USA Contributions: (I) Conception and design: B O’Neil, OB Gbolahan, M Pyko; (II) Administrative support: None; (III) Provision of study material or patients: None; (IV) Collection and assembly of data: OB Gbolahan, M Pyko, MA Schact, EW Beckley, TP Laroche; (V) Data analysis and interpretation: OB Gbolahan, M Pyko, MA Schact, EW Beckley, TP Laroche; (VI) Manuscript writing: All authors; (VII) Final approval of manuscript: All authors.

Correspondence to: Bert O’Neil, MD. Oncology, Indiana Cancer Pavilion, Indiana University School of Medicine, 535 Barnhill Drive, Indianapolis, IN 46202-5289, USA. Email: bhoneil@iu.edu.

\begin{abstract}
The management of hepatocellular carcinoma (HCC) remains challenging due to late presentation and the presence of accompanying liver dysfunction. As such, most patients are not eligible for curative resection and liver transplant. Management in this scenario depends on a number of factors including hepatic function, tumor burden, patency of hepatic vasculature and patients' functional status. Based on these, patients can be offered catheter based intra-arterial therapy for intermediate stage disease and in more advanced disease, sorafenib. Given recent data, regorafenib is now an option following failure of sorafenib. Catheter directed intra-arterial therapy takes advantage of tumor hypervascularity and the unique dual blood supply of the liver, as hepatic tumors receive arterial perfusion via the hepatic artery while the rest of the liver is supplied by the portal vein. This allows selective embolization and delivery of chemotherapeutic agents to the tumor. Compared to best supportive care, intra-arterial therapy offers a survival benefit in intermediate stage HCC and is the recommended approach for treatment. None of the catheter based approaches; including bland embolization, conventional trans-arterial chemoembolization (cTACE), drug eluting bead trans-arterial chemoembolization (DEB-TACE) or trans-arterial radioembolization (TARE) offers a clear advantage over the other, although DEB-TACE may be characterized by less systemic toxicity. All of these approaches are contraindicated in patients with portal vein thrombosis (PVT). On the other hand, intraarterial, radio embolization, with Yttrium-90 (Y90) can be offered to patients with PVT. The place of this modality in management of HCC is still being investigated. The role of sorafenib in advanced HCC is not in doubt, as until recently, it was the only systemic therapy approved for the management in this setting. This is despite multiple trials evaluating other agents. The addition of sorafenib to catheter-based therapy in intermediate stage disease has also failed to show any benefit. The modest survival benefit with sorafenib and the failure of other targeted agents suggest that it is important to look beyond inhibition of angiogenesis in advanced HCC. Identification of key drivers and mediators of HCC remains paramount for successful drug development. In line with this, it is refreshing that the excitement that has followed developments in cancer immunotherapy is finding its way to HCC with early trials of anti-PD1 monoclonal antibodies showing sufficient activity that phase III trials are now ongoing for Pembrolizumab and Nivolumab in advanced HCC. Future drug development efforts will focus on defining the feasibility of combining different treatment approaches targeting multiple important modulators of HCC.
\end{abstract}

Keywords: Catheter directed therapy; chemoembolization; locoregional; regorafenib; sorafenib; hepatocellular cancer

Submitted Jan 15, 2017. Accepted for publication Mar 06, 2017.

doi: 10.21037/jgo.2017.03.13

View this article at: http://dx.doi.org/10.21037/jgo.2017.03.13 


\section{Introduction}

Hepatocellular carcinoma (HCC) is the most common primary liver malignancy, accounting for $90 \%$ of the primary liver cancers in the United States (1). Hepatic fibrosis and cirrhosis are the most common precursors to the development of HCC. HCC is closely associated with chronic hepatitis C and hepatitis B infections. Hepatitis C is more common in the United States, affecting over 3 million people; however, worldwide Hepatitis B is a more common infection. In developed countries, alcohol induced liver disease is recognized as a leading cause of HCC. Historically HCC had a very poor prognosis, which was in part due to advanced stage of disease at diagnosis. With implementation of screening programs for at risk individuals, more early stage disease was found and outcomes improved with survival rates doubling between 1992 and 2004 (1).

The definitive therapies for HCC remain surgical resection, ablation or liver transplantation (2); however, most patients with HCC are ineligible for these therapies due either to disease burden (for transplant and ablation) or severity of liver disease (for surgical resection). Multiple classification schemes are available to stratify patients with preserved hepatic function or poor hepatic function in an effort to determine which therapies they can undergo. The Barcelona Clinic Liver Cancer Staging (BCLC) system is the most widely used and takes into account hepatic function, extent of tumor involvement, and performance status (3). The BCLC system classifies HCC into five stages based on tumor burden, Child-Pugh score, and Eastern Cooperative Oncology Group (ECOG) performance status (4). Surgical resection is considered in patients with preserved hepatic function, normal bilirubin, and no portal hypertension (BCLC stage 0 ). If patients are not candidates for resection they may be considered for liver transplantation or percutaneous ablative therapies if they have a solitary tumor less than $5 \mathrm{~cm}$ or three tumors, each less than $3 \mathrm{~cm}$ (BCLC stage 0 and A). Percutaneous therapies such as thermal ablation (cryotherapy, microwave ablation, and radiofrequency ablation) or chemical ablation can be used as an alternative to surgery (BCLC stage A) (5). The remaining patients may be considered for transarterial therapies (5) Sorafenib, a multikinase inhibitor is the only systemic therapy that has shown a survival benefit for patients with HCC (6).

In this article, we will discuss the range of treatments, both locoregional and systemic, that is available to treat HCC that is not amenable to curative therapy (BCLC B and C). For the most part the goal of these treatments is prolongation of life and palliation of symptoms rather than cure.

\section{Loco-regional therapy for HCC}

There are multiple transarterial therapies available based on the tumor size and characteristics as well as the patient's clinical status. These therapies include transarterial embolization (TAE), conventional transarterial chemoembolization (cTACE), drug eluting bead (DEB) transarterial chemoembolization (DEB-TACE), and transarterial radioembolization (TARE). The rationale for catheter directed local regional therapies in the treatment of HCC is predicated on the fact that there is a mismatch between blood supply to normal hepatic parenchyma and HCC lesions. Normal liver parenchyma receives $3 / 4$ of its blood supply from the portal vein with the remaining $1 / 4$ arriving via the hepatic artery. In contrast, advanced HCC derives the bulk of its blood supply from the hepatic artery (7). cTACE and DEB-TACE take advantage of that difference in blood supply, allowing for greater delivery of embolic agent to the tumor while minimizing insult to normal hepatocytes supplied by the same hepatic artery (3).

Transarterial therapy not only provides a survival benefit $(8-11)$, in select cases therapies can be used to downstage a patient's tumor burden. The goal of tumor reduction is to place the patient back within Milan/UCSF Criteria so they may undergo liver transplantation, ultimately offering them a chance at a curative therapy. cTACE and DEB-TACE are contraindicated in patients with advanced cirrhosis (Child-Pugh C) and poor functional status, while tumor within the main portal vein, biliary obstruction and hepatic encephalopathy are considered relative contraindications.

\section{Embolic therapies in the HCC treatment algorithm}

While several staging systems and treatment algorithms exist for HCC $(5,12)$, the most widely reported and accepted in the literature is the Barcelona Clinic Liver Cancer Staging Classification and Treatment Algorithm (BCLC) (13). Patients classified as intermediate stage B demonstrate multinodular disease outside of transplantable criteria with good performance status. These patients are typically considered for transarterial treatment options, the most common form being TACE.

\section{TAE}

TAE involves the endovascular occlusion of arteries 
supplying a targeted tumor. TAE has been performed for over three decades in the treatment of HCC (14). This form of embolization is typically completed with gelatin sponge particles, spherical embolic agents or PVA particles alone, and is often referred to as "bland" embolization. While specific techniques and materials are quite variable, the basic goal is induced cell death by severe hypoxia achieved via occlusion vascular supplies to the tumor.

\section{Patient selection}

TAE may be considered in BCLC class B patients and is limited in patients with class $\mathrm{C}$ disease. While TACE carries a $1 \mathrm{~A}$ recommendation for stage $\mathrm{B}$ disease (15), some institutions rely on bland embolization due to the overall cost versus TACE as well as conflicting studies comparing their efficacy $(14,16-18)$. Age has not been demonstrated as a factor that impacts outcome with TAE (19). Importantly, no definitive exclusion criteria based on laboratory values exists for TAE treatment; however, advanced cirrhosis, elevated total bilirubin $>4 \mathrm{mg} / \mathrm{dL}$, serum creatinine $>2 \mathrm{mg} / \mathrm{dL}$, portal vein involvement and hepatic encephalopathy are considered relative contraindications.

\section{Technique and considerations}

Arterial access is obtained and catheter and microcatheter combinations are used to select the hepatic arterial branches supplying a tumor. From that location, embolic agents are infused until stasis of flow is seen in the selected artery. The goal of catheter placement is to ensure complete vascular coverage of the tumor with sparing of as much normal parenchyma as possible, limiting the amount of normal tissue ischemia and post embolization syndrome. Embolic agents selection and composition varies, but small embolic material sizes generally range from 40-300 $\mu \mathrm{m}$ (15). One important consideration in catheter directed embolotherapy involves intra-tumoral arterial-venous shunts, which may result in inadvertent pulmonary arterial embolization if particle size is too small.

Post embolization syndrome may be experienced in patients and is typically worse in those requiring larger territorial embolization. This is usually self-limited and may present with abdominal pain, nausea, vomiting, ileus, fatigue and/or fever within the first 72 hours post-embolization (20). For that reason, patients treated with both TAE and TACE are commonly admitted overnight to the hospital for pain control prior to discharge, usually the following morning. Post-procedure imaging and laboratory assessment are typically completed 4-6 weeks after treatment to assess response. Treatment may require multiple sessions if additional vascularity or progression of disease is noted on post procedure imaging.

\section{Outcomes}

Studies have demonstrated survival benefit with TACE relative to supportive care. No such comparative data exist for TAE; some studies have demonstrated inferior survival response with TAE (16). While meta-analysis data suggesting non-inferiority of TAE relative to TACE has been published (14), a recent prospective trial from 2009 comparing DEB-TACE with TAE showed longer time to progression (TTP) and a greater percentage of individuals with complete response in the DEB-TACE group (17). More recently, a study was published demonstrating no difference in tumor response, progression free survival (PFS) or overall survival (OS) when comparing bland embolization with DEB embolization (18). As such, TAE and TACE may be regarded as relatively equal in their management of intermediate stage HCC, with definitive studies unlikely to be completed. Major complications of TAE are similar to TACE therapy (discussed below) and include liver failure, vascular injury, sepsis, non-target embolization, abscess/ biloma formation, and bleeding secondary to arterial access.

\section{TACE}

As the name suggests, chemoembolization employs a dual mechanism of action that results in a synergistic tumoricidal effect. The embolic agent induces ischemia and local hypoxia within the treated tissue. The intraarterial infusion of the chemotherapy agent delivers higher drug concentrations to the tumor than would otherwise be tolerated by the patient via a systemic route (21). The hypoxia induces secretion of vascular endothelial growth factor (VEGF), which results in increased vessel permeability (22). Leaking vessels in combination with hypoxia induce cell membrane dysfunction and stasis of blood flow leads to higher intracellular deposition of the chemotherapy agent and greater intra-hepatic retention. These changes result in less of the administered chemotherapy agent making it into the systemic circulation thus decreasing side effects and toxicity (8). 


\section{Patient selection}

cTACE and DEB-TACE may have a select role as a second line treatment in early stage A patients who have recurrence after failing an above mentioned first line therapy. For intermediate stage $\mathrm{B}$ patients, those with multinodular HCC, a performance status of 0 and preserved liver function (Child-Pugh A), cTACE and DEB-TACE are considered first line therapies (21) with a $1 \mathrm{~A}$ recommendation supporting their utilization (23). Select patients with elevated liver function, mildly impaired performance statuses with or without vascular invasion can be treated; however, the incidence of complications including liver failure increase in these populations. cTACE and DEB-TACE are contraindicated in patients with advanced cirrhosis (Child-Pugh C), performance status $>2$, significant extrahepatic tumor burden and patient's with medically refractory hepatic encephalopathy. Relative contraindications include tumor within the main portal vein, biliary obstruction, total bilirubin $>4$, serum creatinine $>2$, and significant arteriovenous tumor shunting.

\section{Technique}

Overall technical performance of TACE is similar to TAE in practice in which a catheter and microcatheter system are negotiated into the hepatic arterial tree or accessory branches which feed the tumor. The goal of catheter placement during treatment is to achieve complete territorial coverage of the tumor vascularity sparing as much normal parenchyma as possible for delivery of chemotherapeutic embolization beads. Additionally, some institutions are performing TACE in conjunction with ablation as curative therapy (24), with one study demonstrating improved outcomes in single large HCCs $(>3 \mathrm{~cm}$ ) when compared to DEB-TACE alone (25).

\section{CTACE}

In cTACE, lipiodol (a poppy seed oil derivative) is utilized as the carrying embolic agent used to deliver the desired chemotherapy drug to the intended target. The most commonly used chemotherapy agents are cisplatin, doxorubicin, epirubicin and mitomycin C (26). The plethora of options available for concocting chemoembolic mixtures has resulted in significant variation in treatment protocols from center to center, making standardization of cTACE difficult. The formulated lipiodol chemotherapy solution is then administered through the catheter within the selected hepatic arterial distribution supplying the HCC lesion(s). Upon completion of the chemoembolic infusion and without change in catheter position, bland particles may be injected further decreasing arterial blood flow in the treated section of liver. That additional step is thought to improve the chemotherapy dwell time, hereby increasing tumor intracellular uptake and decreasing washout into the systemic circulation.

\section{DEB-TACE}

DEB-TACE represents the second iteration of cTACE where the liquid carrier embolic agent, lipiodol, has been replaced with microspheres ranging in size from approximately $45-300 \mu \mathrm{m}$ with some institutions opting for larger particles. These microspheres are impregnated with a chemotherapy agent, most often with doxorubicin in the United States (27). As with all catheter directed local regional therapies, a catheter is positioned within the desired hepatic artery arterial distribution supplying the HCC lesion(s) through which the drug eluding beads are infused. The goal is to achieve near stasis of blood flow within the treated hepatic artery distribution at the end of infusion (28). Relative to cTACE, there is data to suggest DEB-TACE may result in longer tissue dwell times by the chemotherapy agent, ultimately increasing exposure of the targeted HCC lesion $(29,30)$.

\section{Outcomes}

Response of HCC to chemoembolization is assessed on follow up imaging and is most often graded using the modified Response Evaluation Criteria in Solid Tumors (mRECIST) (31). Modified RECIST is preferred over RECIST 1.1 in evaluating response to treatment for liver lesions because RECIST assesses response to chemotherapeutic agents with changes in tumor size as the only contributing criteria it may be misleading when applied to targeted therapies (e.g., sorafenib) or interventional therapies. Due to mRECIST assessing for arterial phase enhancement it is more suited in assessing patients who have received targeted or interventional therapies. It is not entirely uncommon to demonstrate incomplete response or no response to therapy on follow up imaging after initial treatment of the HCC lesions; however, it has been demonstrated that failure to achieve adequate response after an initial round of chemoembolization is not an accurate predictor of poor tumor response to subsequent chemoembolization (32). 
TACE induced acute liver failure as defined by an increase in Child-Pugh score $\geq 2$, elevation of total serum bilirubin by $\geq 2 \mathrm{mg} / \mathrm{dL}$, or new hepatic encephalopathy or ascites occurring within 2 weeks of therapy is reported at a rate between $2 \%$ to $20 \%(33-35)$. Mild post procedure elevation of liver function tests is expected given the ischemic insult that occurs to normal liver parenchyma supplied by the same hepatic artery distribution as the targeted tumor. Major complications (vascular injury, sepsis, non-target embolization, abscess/biloma formation, biliary stricture or variceal bleeding) related to TACE occur in approximately $5 \%$ of cases with a $1 \%$ risk of death $(33,36)$.

As seen in TAE, post embolization syndrome (abdominal pain, nausea, vomiting, ileus, fatigue and/or fever) is the most commonly reported adverse event associated with chemoembolization of HCC. It is experienced to some degree in up to $90 \%$ of cases (37) with the majority of symptoms markedly improving with 48-72 hours.

Meta-analysis evaluating the effect of cTACE on OS when compared to conservative therapy demonstrated cTACE imparts a significant survival benefit to patients with an improvement in a 2-year survival ranging between $20 \%$ to $60 \%$ (38) and improve median survival from 16 to 20 months (39). DEB-TACE has also been demonstrated to have similar significant survival benefits, however recent head to head comparison of cTACE to DEB-TACE has failed to demonstrate a superior therapy when OS is the endpoint. The PRECISION V study did demonstrate a superior safety profile with DEB-TACE with significant reduction in the observed doxorubicin related side effects and serious liver toxicity when compared to cTACE (8). Patients treated with DEB-TACE required a significantly lower number of treatments which enhance the safety profile and lead to cost savings (40).

\section{TARE with Yttrium-90 (Y90)}

TARE (41) relies on the intraarterial delivery of radioactive spheres to the vascular beds of tumor. Y90 is a pure beta emitter with a half-life of 2.67 days and tissue penetration of up to approximately $11 \mathrm{~mm}$ with a mean of $2.5 \mathrm{~mm}$. It is loaded on the surface of resin microspheres (SIR-Spheres, Sirtex Medical, Australia) or intrinsic to the glass matrix of microspheres (TheraSphere, Nordion, Canada), which range in size between 20-30 $\mu \mathrm{m}$ (Therasphere) and 20-60 $\mu \mathrm{m}$ (SIR-Spheres). The dominant antitumor effect of Y90 is induced by radiation as opposed to drug and ischemic effects as observed with TACE (42).

\section{Patient selection}

TARE using Y90 is a recognized treatment option with a palliative role in the treatment of unresectable HCC $(43,44)$. Although BCLC guidelines suggest transarterial chemoembolization (TACE) is the standard of care for intermediate stage (BCLC B) disease, there is growing evidence for the role of $\mathrm{Y} 90$ in the treatment of these patients as well as those with either early (BCLC A) or advanced stage (BCLC C) disease $(45,46)$. Importantly, Y90 has been shown to be safe in those patients with portal vein thrombosis (PVT) due to the reliance on delivery of radiation over blockade of vascularity as with TAE and TACE (47). Patients with Child-Pugh A (with/ without PVT) or Child-Pugh B (without PVT) disease staging are most likely to benefit from Y90 (44). Typical inclusion criteria for treatment of liver disease with Y90 include total bilirubin $<2.0 \mathrm{mg} / \mathrm{dL}$ with ECOG score $0-2$. Relative contraindications include renal failure and untreated portosystemic varices at risk of bleeding. Absolute contraindications include decompensated cirrhosis, tumor burden $>75 \%$ of liver parenchyma, and uncorrectable shunts to either the lungs or gastrointestinal tract (45).

\section{Technical considerations}

Y90 is an intra-arterial radiation therapy that allows for much higher doses of radiation to be delivered to the diseased liver when compared to external beam radiation, which is limited by the radiosensitivity of normal liver parenchyma (48). Delivery of Y90 via the hepatic artery allows for preferential delivery of radiation to the tumors while minimizing impact on normal parenchyma due to the hypervascular nature of HCC.

Y90 radioembolization is typically performed in a twostage manner. The first stage of the procedure involves an outpatient planning angiogram performed 1-2 weeks prior to treatment. Planning angiography provides the opportunity for vascular mapping, embolization of variant vascularity to ensure safe treatment, as well as evaluation of tumor shunting which would result in inadvertent radioactivity delivery to the lungs. In order to evaluate tumor shunting, technetium-99 m labeled macroaggregated albumin (MAA) is injected into the desired hepatic lobar or segmental artery followed by planar/SPECT gamma imaging to determine the fraction of shunting from the liver to the lungs (41).

The treatment phase, again performed on an outpatient 
basis, involves delivery of $\mathrm{Y} 90$ in a lobar, segmental or sub-segmental fashion. Whole-liver therapy is generally avoided due to the increased risk of liver failure. Antibiotic, anti-emetic, and pain control medications are usually unnecessary following radioembolization. Responses to therapy may take up to 3-6 months on imaging (49) and therefore, typical clinic follow up is performed at 3 months intervals following treatment at which time biochemical and imaging follow up is obtained (44). One study demonstrated diffusion weighted imaging $(9,50)$ at 1 month preceded anatomic size changes at 3 months post $\mathrm{Y} 90$ (43).

In addition to traditional Y90 administration in a staged lobar fashion, more novel treatment concepts are actively being investigated and include radiation "segmentectomy" and "lobectomy" as well as downstaging to liver transplantation (45).

\section{Outcomes}

Response rate, OS, and TTP following Y90 radioembolization vary depending on a number of factors, most importantly baseline patient stage (BCLC, UNOS, Child-Pugh). Sangro et al. demonstrated Median OS following radioembolization to be 24.4 months (BCLC A), 16.9 months (BCLC B), and 10.0 months (BCLC C). The most significant prognostic factors for survival upon multivariate analysis included ECOG status, tumor burden ( $>5$ nodules), an INR $>1.2$ and extrahepatic disease (51). TTP was longer for patients with Child-Pugh A and B without PVT, 15.5 and 13 months, respectively while patients with PVT demonstrated 5.6 and 5.9 months, respectively. Median OS in patients without PVT has been demonstrated to be 17.3 (45) and 18.4 (51) months in Child-Pugh A and 13.5 months (45) in Child-Pugh B patients. Hilgard et al. demonstrated an overall TTP of 10.0 with 8.0 and 11.8 months in patients with and without PVT respectively. Median OS of 16.4 months in patients without PVT while portal vein involvement dropped median OS to 10.0 months (11).

The most common clinical toxicities include fatigue (57\%), pain (23\%), and nausea/vomiting (20\%). Additionally, radioembolization-induced liver disease (REILD) is a syndrome that may appear 4-8 weeks following radioembolization manifesting as jaundice, ascites, and an increase in gamma-glutamyl transpeptidase/alkaline phosphatase in non-cirrhotic patients $(45,52)$.

Although no survival benefit over TACE has yet to be demonstrated, radioembolization has been shown to provide secondary benefits over TACE including decreased toxicity (44) and improved quality-of-life regarding diarrhea, fever, chills adverse effects and anorexia (53). Furthermore, radioembolization can be performed on an outpatient basis. Given these factors $\mathrm{Y} 90$ is gaining acceptance as a treatment for late BCLC B (intermediate stage) and early BCLC C (advanced stage) disease $(51,54)$.

\section{Systemic therapy for advanced hepatocellular cancer}

\section{Selection of patients for systemic therapy}

Patients meeting the definition of BCLC stage C are considered most optimal for systemic therapy. Within this group, however, lies a large range of clinical variables. As such, choices between locoregional and systemic therapy can be difficult (such as in a patient with a relatively large tumor who also has tumor-associated main or main branch PVT. There is little argument that this modality is a reasonable choice for patients with extrahepatic metastatic disease, or those whose tumors have progressed after one or more locoregional treatments.

Early attempts at management focused on traditional cytotoxic chemotherapy, particularly doxorubicin (47). However, HCC has long been recognized as chemoresistant, with very low radiographic response rates to chemotherapy in modern trials $(55,56)$. Thus to date, no single chemotherapy agent or combination of agents has led to a significant increase in OS $(57,58)$. This has led research away from chemotherapy to targeted molecular antagonists of pathways thought to be critical to the pathogenesis of this disease. A milestone along that road has been the approval of sorafenib for the management of advanced HCC.

\section{First line treatment}

Similar to many other solid tumors, angiogenesis is important for the maintenance and growth of HCC. Sorafenib is a small molecule inhibitor of signaling pathways implicated in angiogenesis. Although initially characterized as a Raf-1 and B-RAF inhibitor, it also abrogates phosphorylation and activation of tyrosine kinase domains of the vascular endothelial growth factor receptor (VEGFR), platelet derived growth factor (PDGFR), c-kit and flt1 (59). It was approved for the management of advanced HCC following improvement in OS reported by the SHARP trial (6). In this trial, 602 patients were randomized to sorafenib $400 \mathrm{mg}$ twice daily or placebo. Eighty-two percent of the patients had advanced disease based on the Barcelona 
Clinic Liver Cancer (BCLC) staging and $92 \%$ had an ECOG performance status of 1 and below. Only $5 \%$ of patients in the sorafenib arm had child pugh class B liver dysfunction with the rest falling into $\mathrm{CP}$ class A category with relatively well-preserved liver function. Median OS was 10.7 months with sorafenib and 7.9 months with placebo (HR: $0.69,95 \%$ CI: $0.55-0.87$ ) with an $11 \%$ improvement in survival at 1 year $(44 \%$ vs. $33 \%$ in the placebo arm). This result was complemented by similar findings of improved OS in the Asia-Pacific region (60). Cheng and colleagues reported an OS benefit improvement of about 2 months with sorafenib over placebo (6.5 vs. 4.2 months, HR: 0.68, 95\% CI: 0.5-0.97). This study enrolled 271 patients and assigned them in a 2:1 ratio to sorafenib and placebo. The difference in survival between the two studies has been attributed to the higher prevalence of patients with more advanced disease in the Asia-Pacific trial, with $95 \%$ of patients in this trial having Stage C BCLC HCC, compared to $82 \%$ in the SHARP trial. Also, $68 \%$ (vs. $53 \%$ ) of patients had extra-hepatic spread of HCC. This is worth mentioning because extra hepatic spread was one of the sub-groups analyzed in the SHARP trial where sorafenib did not conclusively show a benefit. More importantly, the preponderance of hepatitis B virus (HBV) related disease in Asia-Pacific has been argued to be a reason for the relatively low survival rates as $\mathrm{HBV}$ related HCC has a more aggressive course with worse prognosis than hepatitis $\mathrm{C}$ virus $(\mathrm{HCV})$ related disease which is prevalent in Western Europe and the United States. In keeping with this, in a sub-group analysis of the SHARP trial the small number of patients with HBV had shorter OS numbers compared to those with HCV related HCC (61).

Despite the relatively modest benefit conferred by sorafenib (and the attendant hope that other agents could reproduce or improve upon this success), there has been little progress with targeted therapy in almost a decade of subsequent drug development. A single arm phase II trial of bevacizumab, the prototypical angiogenesis inhibiting monoclonal antibody directed at VEGF appeared to show a strong signal for further development, with a median PFS of 6.9 months and an OS of 12.4 months among 46 patients (62). Despite this, there has been no phase III trial to confirm this finding, with the field shifting towards combining bevacizumab with other agents similar to other malignancies (63).

Brivanib is a dual kinase domain inhibitor of VEGFR and fibroblast growth factor receptor (FGFR). In addition to its proliferative effects, FGF may play a role in tumor invasion
$(64,65)$ and in overcoming resistance to VEGFR targeted therapy (66). The BRISK-FL study was a phase III noninferiority head to head study of brivanib and sorafenib in the first line setting (67). Despite similar median OS numbers of 9.9 months for sorafenib and 9.5 months for brivanib (HR: 1.06, 95\% CI: 0.93-1.22), the study failed to meet the pre-specified upper limit hazard ratio for non-inferiority of HR less than or equal to 1.08. Brivanib was also less tolerable than sorafenib with the rate of drug cessation secondary to adverse events at $44 \%$ with brivanib and 33\% with sorafenib. Sunitinib another multikinase inhibitor with similar targets to sorafenib, but with a stronger affinity for the VEGFR fared worse compared with sorafenib (68). Not only was OS shorter (7.9 vs. 10.2 months, HR: 1.2), it was significantly more toxic, with Grade 5 treatment related adverse events reported in 17 patients compared to 2 in the sorafenib arm. Similarly, linifanib did not show a benefit over sorafenib (69).

A common motif in the above investigations is that the studies have been based on agents that affect signaling of key angiogenesis receptor pathways. In line with the American Association for the Study of Liver Disease (AASLD) recommendations (70), investigators in the SEARCH trial (71), went in a different direction by attempting to antagonize a separate receptor pathway in addition to angiogenesis blockade. They compared the addition of erlotinib, an epidermal growth factor receptor (EGFR) tyrosine kinase inhibitor (72) and sorafenib to sorafenib only. Despite the compelling rationale, the combination failed to scale the bar. There was a significant difference in disease control rates (DCR) in both arms, with the combination arm faring worse $(43.9 \%$ vs. $52.5 \% \mathrm{P}=0.021)$. Median OS was similar in both arms at 9.5 months with combination compared to 8.5 months with sorafenib only (HR: $0.93,95 \%$ CI: 0.781-1.106). Other agents tried in the first line setting have been similarly underwhelming as noted in Table 1 .

So why have all these agents performed so poorly? A number of reasons have been put forward, chief among which are the clinical and molecular heterogeneity of HCC. As mentioned previously, patients with HBV related HCC have a more aggressive phenotype with worse prognosis than patients with HCV related disease. Importantly, the molecular pathogenesis of HCC is still being worked out. Similar to other solid tumors, there appears to be many more passenger mutations than driver mutations in human HCCs, and in instances where targeted therapies have been most successful, they have targeted critical driver mutations. A few driver gene mutations have been defined in HCC but the dominant driver remains unclear $(77,78)$. Presumably, 
Table 1 Completed phase III clinical trials of targeted therapy in advanced hepatocellular cancer

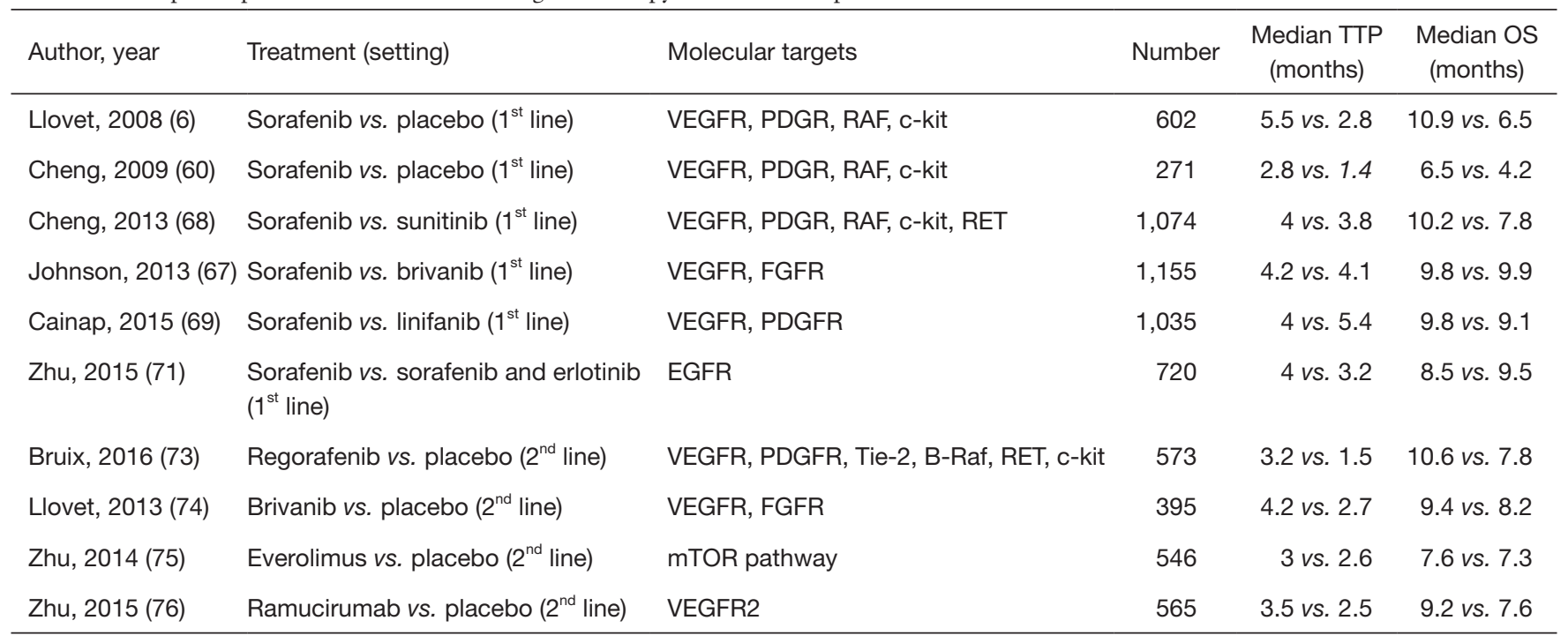

VEGFR, vascular endothelial growth factor receptor; PDGR, platelet derived growth factor receptor; RAF, rapidly accelerated fibrosarcoma; RET, rearranged during transfection,; FGFR, fibroblast growth factor receptor; EGFR, epidermal growth factor receptor; mTOR, mammalian target of rapamycin.

staving off only one of these drivers would be insufficient to significantly check disease progression.

\section{Sorafenib-refractory setting}

In spite of a relatively large number of phase III trial "attempts", no drug has been approved in the second line setting for advanced HCC. As in studies in the first line setting, agents targeted against angiogenic kinases have played a lead role in the sorafenib-refractory setting. Two large, phase III randomized studies, EVOLVE-1 and BRISK-PS failed to show benefit of targeted treatment in this setting. In the EVOLVE-1 trial (75), everolimus, an inhibitor of the mammalian target of rapamycin (mTOR) pathway (79) delivered an OS of 7.6 months compared to 7.3 months for placebo (HR: 1.11, 95\% CI: 0.91-1.36). The authors noted that the time to deterioration in physical functioning was shorter with everolimus. Despite better overall numbers-superior overall response rates (ORR) of $10 \%$ vs. $2 \%$ (OR: $5.4, \mathrm{P}=0.003$ ) and DCR of $61 \%$ vs. $40 \%$ (OR: 2.38, $\mathrm{P}=0.001$ ) -in the BRISK-PS (74) study, brivanib also ultimately failed to show a significant OS benefit compared to placebo [9.4 vs. 8.2 months (HR: $0.85,95 \%$ CI: 0.69-1.15)]. The story was similar with Ramucirumab (76). Curiously, in these second line studies, survival in the placebo arms were similar to the survival in the placebo arm of the SHARP trial of Sorafenib in the 1st line setting suggesting that patients with well-preserved liver function and/or less aggressive cancers were enrolled in these trials. The mRECIST (31) criteria for assessing response to therapy was published after the SHARP trial to account for the radiologic "pseudo-progression" secondary to tumor necrosis and bleeding which may be construed as treatment failure despite continued clinical benefit from sorafenib. Some patients with radiologic progression on sorafenib despite clinical stability may have been enrolled into these studies, as modified RECIST has not been universally adopted.

On a more positive note, recently, the results of the phase III RESORCE trial of regorafenib, another multikinase inhibitor, targeting the VEGFR among others was recently published. The authors reported an OS advantage over placebo (OS: 10.6 vs. 7.8 months, HR: 0.63, $95 \%$ CI: 0.50-0.79) (73) and this finding is likely to change the management landscape of sorafenib refractory HCC. Interesting findings have also been reported in a phase II trial of tivantinib a c-MET inhibitor. Although there was no clinically meaningful difference in the primary end point of TTP of 1.6 vs. 1.4 months (HR: 0.64, 90\% CI: 0.43-0.94), and other end points including PFS and OS, between tivantinib and placebo, pre-planned subgroup analysis revealed a doubling of TTP with tivantinib in a c-MET high group compared to placebo (2.7 vs. 1.4 months, HR: 0.43, 95\% CI: 0.19-0.97) (80). This has prompted a phase III trial 
of tivantinib compared to placebo in c-MET high HCC.

\section{Loco-regional therapy and sorafenib}

TACE is the most commonly employed option for locoregional therapy, with multiple guidelines endorsing this approach in intermediate stage HCC $(13,81)$. Despite the significant improvement in TTP and OS with TACE, BCLC stage B HCC is largely incurable. Cytotoxicity and disease control are engendered in the short term, but TACE ultimately promotes angiogenesis on account of the hypoxia induced by embolization. This leads to up regulation of HIF-1alpha, and eventual production of angiogenic factors particularly VEGF (82). The rationale for combining sorafenib with catheter-based treatment in HCC is to counteract this paradoxical angiogenic effect with the hope of extending clinical benefit derived from TACE. This has however, not been borne out in clinical trials.

Among Japanese and Korean patients with intermediate stage HCC who had at least $25 \%$ tumor shrinkage with TACE, subsequent administration of sorafenib did not provide additional benefit in terms of OS or TTP (83). Others have focused on concurrent administration of sorafenib and TACE with multiple studies confirming the safety of this approach (84-86). These studies also suggested interesting efficacy numbers in terms of OS and TTP although they were mostly single arm studies and not powered to determine such an effect. The SPACE trial is a phase II trial that randomized 307 patients to TACE-DEB with sorafenib and TACE-DEB with placebo (87). There was no difference in the primary end point of TTP in both arms at 169 vs. 166 days in the sorafenib arm compared to placebo (HR: 0.797 ; 95\% CI: 0.588-1.080, $\mathrm{P}=0.072$ ). A major problem with this study was that in close to a third of patients randomized, initially described target lesions were not confirmed on central radiological review. More importantly, a similar fraction received only one round of TACE on account of adverse events, which may very well have been reversible allowing the patients to undergo and possibly benefit from further therapy. It remains unclear why the combination of TACE and sorafenib has not been as beneficial as expected, but some experts speculate that patients with more advanced disease are more likely to benefit from this combined approach than those with intermediate stage disease (88). The GIDEON study suggests this (89), but this is registry data with only a small number of reports of concurrent TACE and sorafenib. At this time, there is no high quality evidence to suggest that adding sorafenib to a catheter- based treatment modality is beneficial.

\section{Novel options and future perspectives}

Although it appears that therapy directed at angiogenesis and VEGFR signaling may have hit a ceiling, it is encouraging that drug development for advanced HCC continues to explore new avenues. Immunotherapy with immune checkpoint blockade is undoubtedly one of the most exciting areas in cancer therapeutics in the last few years, with significant clinical activity reported with CTLA-4 and PD-1 blocking agents, particularly in melanoma (90) and non-small cell lung cancer (NSCLC) $(91,92)$. This has significantly and positively impacted outcomes in these diseases. In line with results from other solid tumors, investigators are reporting promising activity in HCC also. A phase II trial of nivolumab, an anti PD1 monoclonal antibody, was reported at the recent American Society of Clinical Oncology (ASCO) annual meeting (93). Investigators reported that nivolumab was tolerable, with grade 3-4 adverse events reported in $20 \%$ of 51 patients, most commonly elevations in liver enzymes. Median OS in this small study was 15.1 months (95\% CI: 9.6-28.6 months). Importantly close to half of the patients were still alive after 18 months. We note that enrolled patients had relatively well-preserved liver function with CPS grade A. A phase III trial comparing nivolumab to sorafenib is already recruiting participants comparing nivolumab to sorafenib in the first line setting (94). A phase III trial of pembrolizumab, another PD-1 inhibitor is also recruiting patients who have advanced on sorafenib (95) and other immunotherapy options including cancer vaccines and immune stimulators are in various stages of drug development.

\section{Discussion and summary}

HCC is well recognized as an aggressive tumor and for those patients who are not candidates for curative therapy transarterial and systemic therapies offer a good alternative in slowing tumor progression. With the wide variety of treatment modalities, a multidisciplinary approach to therapeutic considerations is recommended. While other modalities offer curative therapies in limited disease, the majority of cases presents beyond early stage disease and receive catheter directed therapy for downstaging of tumor burden or as definitive therapy. When deciding between the available transarterial therapies it is important to 
realize that no clear evidence suggesting one therapy over another exists however many factors must be considered including institutional availability, physician skill-set and training, inpatient versus outpatient therapy, as well as cost. When it comes to transarterial therapies, one important consideration that applies to all embolic based therapies, is that blockade of hepatic arteries at a proximal or distal level can lead to collateral vessel formation (96), which may limit the ability to repeat embolization by conventional hepatic vasculature. Formation of collateral vasculature may preclude effective treatment with radioembolization if extrahepatic supply is recruited. Because of this phenomenon treatment with TAE or TACE can preclude effective treatment with Y90. Because much of our knowledge about transcatheter therapies for liver malignancy involves retrospective studies, little data exists offering head to head comparison for available treatment options and more prospective studies and comparative studies between treatments is needed.

In terms of systemic therapies, it is likely that in patients with preserved liver function, combination therapy with agents targeting different driver signaling pathways (and the tumor microenvironment) may lead to further improvement in outcomes in this disease. Combined locoregional and systemic therapies, particularly the immunotherapies, are also early in study and results very much anticipated. To achieve a goal of substantial improvement, however, it is vital that we continue to work out the molecular events that underpin the pathogenesis of HCC.

\section{Acknowledgements}

None.

\section{Footnote}

Conflicts of Interest: Bert O’Neil has consulted for Bayer, Inc.; the other authors have no conflicts of interest to declare.

\section{References}

1. Altekruse SF, McGlynn KA, Reichman ME. Hepatocellular carcinoma incidence, mortality, and survival trends in the United States from 1975 to 2005. J Clin Oncol 2009;27:1485-91.

2. Shrimal A, Prasanth M, Kulkarni AV. Interventional radiological treatment of hepatocellular carcinoma: an update. Indian J Surg 2012;74:91-9.
3. Kumar Y, Sharma P, Bhatt N, et al. Transarterial Therapies for Hepatocellular Carcinoma: a Comprehensive Review with Current Updates and Future Directions. Asian Pacific journal of cancer prevention: Asian Pac J Cancer Prev 2016;17:473-8.

4. Llovet JM, Bru C, Bruix J. Prognosis of hepatocellular carcinoma: the BCLC staging classification. Semin Liver Dis 1999;19:329-38.

5. Yau T, Tang VY, Yao TJ, et al. Development of Hong Kong Liver Cancer staging system with treatment stratification for patients with hepatocellular carcinoma. Gastroenterology 2014;146:1691-700 e3.

6. Llovet JM, Ricci S, Mazzaferro V, et al. Sorafenib in advanced hepatocellular carcinoma. N Engl J Med 2008;359:378-90.

7. Breedis C, Young G. The blood supply of neoplasms in the liver. Am J Pathol 1954;30:969-77.

8. Lammer J, Malagari K, Vogl T, et al. Prospective randomized study of doxorubicin-eluting-bead embolization in the treatment of hepatocellular carcinoma: results of the PRECISION V study. Cardiovasc Intervent Radiol 2010;33:41-52.

9. Maluccio MA, Covey AM, Porat LB, et al. Transcatheter arterial embolization with only particles for the treatment of unresectable hepatocellular carcinoma. J Vasc Interv Radiol 2008;19:862-9.

10. Takayasu K, Arii S, Ikai I, et al. Prospective cohort study of transarterial chemoembolization for unresectable hepatocellular carcinoma in 8510 patients. Gastroenterology 2006;131:461-9.

11. Hilgard P, Hamami M, Fouly AE, et al. Radioembolization with yttrium-90 glass microspheres in hepatocellular carcinoma: European experience on safety and long-term survival. Hepatology 2010;52:1741-9.

12. Kudo M, Chung H, Osaki Y. Prognostic staging system for hepatocellular carcinoma (CLIP score): its value and limitations, and a proposal for a new staging system, the Japan Integrated Staging Score (JIS score). J Gastroenterol 2003;38:207-15.

13. European Association For The Study Of The L, European Organisation For R, Treatment Of C. EASL-EORTC clinical practice guidelines: management of hepatocellular carcinoma. J Hepatol 2012;56:908-43.

14. Marelli L, Stigliano R, Triantos C, et al. Transarterial therapy for hepatocellular carcinoma: which technique is more effective? A systematic review of cohort and randomized studies. Cardiovasc Intervent Radiol 2007;30:6-25. 
15. Shah RP, Brown KT, Sofocleous CT. Arterially directed therapies for hepatocellular carcinoma. AJR Am J Roentgenol 2011;197:W590-602.

16. Llovet JM, Real MI, Montana X, et al. Arterial embolisation or chemoembolisation versus symptomatic treatment in patients with unresectable hepatocellular carcinoma: a randomised controlled trial. Lancet 2002;359:1734-9.

17. Malagari K, Pomoni M, Kelekis A, et al. Prospective randomized comparison of chemoembolization with doxorubicin-eluting beads and bland embolization with BeadBlock for hepatocellular carcinoma. Cardiovasc Intervent Radiol 2010;33:541-51.

18. Brown KT, Do RK, Gonen M, et al. Randomized Trial of Hepatic Artery Embolization for Hepatocellular Carcinoma Using Doxorubicin-Eluting Microspheres Compared With Embolization With Microspheres Alone. J Clin Oncol 2016;34:2046-53.

19. Thornton RH, Covey A, Petre EN, et al. A comparison of outcomes from treating hepatocellular carcinoma by hepatic artery embolization in patients younger or older than 70 years. Cancer 2009;115:5000-6.

20. Castells A, Bruix J, Ayuso C, et al. Transarterial embolization for hepatocellular carcinoma. Antibiotic prophylaxis and clinical meaning of postembolization fever. J Hepatol 1995;22:410-5.

21. Konno T. Targeting cancer chemotherapeutic agents by use of lipiodol contrast medium. Cancer 1990;66:1897-903.

22. Ferrara N, Houck K, Jakeman L, et al. Molecular and biological properties of the vascular endothelial growth factor family of proteins. Endocr Rev 1992;13:18-32.

23. Villanueva A, Hernandez-Gea V, Llovet JM. Medical therapies for hepatocellular carcinoma: a critical view of the evidence. Nat Rev Gastroenterol Hepatol 2013;10:34-42.

24. Iezzi R, Pompili M, Posa A, et al. Combined locoregional treatment of patients with hepatocellular carcinoma: State of the art. World J Gastroenterol 2016;22:1935-42.

25. Iezzi R, Pompili M, La Torre MF, et al. Radiofrequency ablation plus drug-eluting beads transcatheter arterial chemoembolization for the treatment of single large hepatocellular carcinoma. Dig Liver Dis 2015;47:242-8.

26. Crissien AM, Frenette C. Current management of hepatocellular carcinoma. Gastroenterol Hepatol (N Y) 2014;10:153-61.

27. Lewis AL, Gonzalez MV, Lloyd AW, et al. DC bead: in vitro characterization of a drug-delivery device for transarterial chemoembolization. J Vasc Interv Radiol 2006; 17:335-42.
28. Lencioni R, de Baere T, Burrel M, et al. Transcatheter treatment of hepatocellular carcinoma with Doxorubicinloaded DC Bead (DEBDOX): technical recommendations. Cardiovasc Intervent Radiol 2012;35:980-5.

29. Lencioni R. Loco-regional treatment of hepatocellular carcinoma. Hepatology 2010;52:762-73.

30. Namur J, Citron SJ, Sellers MT, et al. Embolization of hepatocellular carcinoma with drug-eluting beads: doxorubicin tissue concentration and distribution in patient liver explants. J Hepatol 2011;55:1332-8.

31. Lencioni R, Llovet JM. Modified RECIST (mRECIST) assessment for hepatocellular carcinoma. Semin Liver Dis 2010;30:52-60.

32. Georgiades C, Geschwind JF, Harrison N, et al. Lack of response after initial chemoembolization for hepatocellular carcinoma: does it predict failure of subsequent treatment? Radiology 2012;265:115-23.

33. Sakamoto I, Aso N, Nagaoki K, et al. Complications associated with transcatheter arterial embolization for hepatic tumors. Radiographics 1998;18:605-19.

34. Huang YS, Chiang JH, Wu JC, et al. Risk of hepatic failure after transcatheter arterial chemoembolization for hepatocellular carcinoma: predictive value of the monoethylglycinexylidide test. Am J Gastroenterol 2002;97:1223-7.

35. Chan AO, Yuen MF, Hui CK, et al. A prospective study regarding the complications of transcatheter intraarterial lipiodol chemoembolization in patients with hepatocellular carcinoma. Cancer 2002;94:1747-52.

36. Clark TW. Complications of hepatic chemoembolization. Semin Intervent Radiol 2006;23:119-25.

37. Leung DA, Goin JE, Sickles C, et al. Determinants of postembolization syndrome after hepatic chemoembolization. J Vasc Interv Radiol 2001;12:321-6.

38. Llovet JM, Bruix J. Systematic review of randomized trials for unresectable hepatocellular carcinoma: Chemoembolization improves survival. Hepatology 2003;37:429-42.

39. Lencioni R, Petruzzi P, Crocetti L. Chemoembolization of hepatocellular carcinoma. Semin Intervent Radiol 2013;30:3-11.

40. Kloeckner R, Weinmann A, Prinz F, et al. Conventional transarterial chemoembolization versus drug-eluting bead transarterial chemoembolization for the treatment of hepatocellular carcinoma. BMC Cancer 2015;15:465.

41. Gaba RC, Zivin SP, Dikopf MS, et al. Characteristics of primary and secondary hepatic malignancies associated with hepatopulmonary shunting. Radiology 2014;271:602-12. 
42. Sato K, Lewandowski RJ, Bui JT, et al. Treatment of unresectable primary and metastatic liver cancer with yttrium-90 microspheres (TheraSphere): assessment of hepatic arterial embolization. Cardiovasc Intervent Radiol 2006;29:522-9.

43. Rhee TK, Naik NK, Deng J, et al. Tumor response after yttrium-90 radioembolization for hepatocellular carcinoma: comparison of diffusion-weighted functional MR imaging with anatomic MR imaging. J Vasc Interv Radiol 2008;19:1180-6.

44. Salem R, Lewandowski RJ, Mulcahy MF, et al. Radioembolization for hepatocellular carcinoma using Yttrium-90 microspheres: a comprehensive report of longterm outcomes. Gastroenterology 2010;138:52-64.

45. Salem R, Mazzaferro V, Sangro B. Yttrium 90 radioembolization for the treatment of hepatocellular carcinoma: biological lessons, current challenges, and clinical perspectives. Hepatology 2013;58:2188-97.

46. Mazzaferro V, Sposito C, Bhoori S, et al. Yttrium-90 radioembolization for intermediate-advanced hepatocellular carcinoma: a phase 2 study. Hepatology 2013;57:1826-37.

47. Nerenstone SR, Ihde DC, Friedman MA. Clinical trials in primary hepatocellular carcinoma: current status and future directions. Cancer Treat Rev 1988;15:1-31.

48. Dawson LA, Normolle D, Balter JM, et al. Analysis of radiation-induced liver disease using the Lyman NTCP model. Int J Radiat Oncol Biol Phys 2002;53:810-21.

49. Lewandowski RJ, Sato KT, Atassi B, et al. Radioembolization with $90 \mathrm{Y}$ microspheres: angiographic and technical considerations. Cardiovasc Intervent Radiol 2007;30:571-92.

50. Goodwin JM, Svensson RU, Lou HJ, et al. An AMPKindependent signaling pathway downstream of the LKB1 tumor suppressor controls Snail1 and metastatic potential. Mol Cell 2014;55:436-50.

51. Sangro B, Carpanese L, Cianni R, et al. Survival after yttrium-90 resin microsphere radioembolization of hepatocellular carcinoma across Barcelona clinic liver cancer stages: a European evaluation. Hepatology 2011;54:868-78.

52. Bruix J, Reig M, Sherman M. Evidence-Based Diagnosis, Staging, and Treatment of Patients With Hepatocellular Carcinoma. Gastroenterology 2016;150:835-53.

53. Salem R, Gilbertsen M, Butt Z, et al. Increased quality of life among hepatocellular carcinoma patients treated with radioembolization, compared with chemoembolization. Clin Gastroenterol Hepatol 2013;11:1358-65.e1.
54. Forner A, Llovet JM, Bruix J. Hepatocellular carcinoma. Lancet 2012;379:1245-55.

55. Kato A, Miyazaki M, Ambiru S, et al. Multidrug resistance gene (MDR-1) expression as a useful prognostic factor in patients with human hepatocellular carcinoma after surgical resection. J Surg Oncol 2001;78:110-5.

56. Huang M, Liu G. The study of innate drug resistance of human hepatocellular carcinoma Bel7402 cell line. Cancer Lett 1999;135:97-105.

57. Gish RG, Porta C, Lazar L, et al. Phase III randomized controlled trial comparing the survival of patients with unresectable hepatocellular carcinoma treated with nolatrexed or doxorubicin. J Clin Oncol 2007;25:3069-75.

58. Yeo W, Mok TS, Zee B, et al. A randomized phase III study of doxorubicin versus cisplatin/interferon alpha2b/doxorubicin/fluorouracil (PIAF) combination chemotherapy for unresectable hepatocellular carcinoma. J Natl Cancer Inst 2005;97:1532-8.

59. Wilhelm SM, Carter C, Tang L, et al. BAY 43-9006 exhibits broad spectrum oral antitumor activity and targets the RAF/MEK/ERK pathway and receptor tyrosine kinases involved in tumor progression and angiogenesis. Cancer Res 2004;64:7099-109.

60. Cheng AL, Kang YK, Chen Z, et al. Efficacy and safety of sorafenib in patients in the Asia-Pacific region with advanced hepatocellular carcinoma: a phase III randomised, double-blind, placebo-controlled trial. Lancet Oncol 2009;10:25-34.

61. Bruix J, Raoul JL, Sherman M, et al. Efficacy and safety of sorafenib in patients with advanced hepatocellular carcinoma: subanalyses of a phase III trial. J Hepatol 2012;57:821-9.

62. Siegel AB, Cohen EI, Ocean A, et al. Phase II trial evaluating the clinical and biologic effects of bevacizumab in unresectable hepatocellular carcinoma. J Clin Oncol 2008;26:2992-8.

63. Fang P, Hu JH, Cheng ZG, et al. Efficacy and safety of bevacizumab for the treatment of advanced hepatocellular carcinoma: a systematic review of phase II trials. PloS One 2012;7:e49717.

64. Ogasawara S, Yano H, Iemura A, et al. Expressions of basic fibroblast growth factor and its receptors and their relationship to proliferation of human hepatocellular carcinoma cell lines. Hepatology 1996;24:198-205.

65. Presta M, Dell'Era P, Mitola S, et al. Fibroblast growth factor/fibroblast growth factor receptor system in angiogenesis. Cytokine Growth Factor Rev 2005;16:159-78.

66. Allen E, Walters IB, Hanahan D. Brivanib, a dual FGF/ 
VEGF inhibitor, is active both first and second line against mouse pancreatic neuroendocrine tumors developing adaptive/evasive resistance to VEGF inhibition. Clin Cancer Res 2011;17:5299-310.

67. Johnson PJ, Qin S, Park JW, et al. Brivanib versus sorafenib as first-line therapy in patients with unresectable, advanced hepatocellular carcinoma: results from the randomized phase III BRISK-FL study. J Clin Oncol 2013;31:3517-24.

68. Cheng AL, Kang YK, Lin DY, et al. Sunitinib versus sorafenib in advanced hepatocellular cancer: results of a randomized phase III trial. J Clin Oncol 2013;31:4067-75.

69. Cainap C, Qin S, Huang WT, et al. Linifanib versus Sorafenib in patients with advanced hepatocellular carcinoma: results of a randomized phase III trial. J Clin Oncol 2015;33:172-9.

70. Llovet JM, Di Bisceglie AM, Bruix J, et al. Design and endpoints of clinical trials in hepatocellular carcinoma. J Natl Cancer Inst 2008;100:698-711.

71. Zhu AX, Rosmorduc O, Evans TR, et al. SEARCH: a phase III, randomized, double-blind, placebo-controlled trial of sorafenib plus erlotinib in patients with advanced hepatocellular carcinoma. J Clin Oncol 2015;33:559-66.

72. Iyer R, Bharthuar A. A review of erlotinib--an oral, selective epidermal growth factor receptor tyrosine kinase inhibitor. Expert Opin Pharmacother 2010;11:311-20.

73. Bruix J, Qin S, Merle P, et al. Regorafenib for patients with hepatocellular carcinoma who progressed on sorafenib treatment (RESORCE): a randomised, double-blind, placebo-controlled, phase 3 trial. Lancet 2017;389:56-66.

74. Llovet JM, Decaens T, Raoul JL, et al. Brivanib in patients with advanced hepatocellular carcinoma who were intolerant to sorafenib or for whom sorafenib failed: results from the randomized phase III BRISK-PS study. J Clin Oncol 2013;31:3509-16.

75. Zhu AX, Kudo M, Assenat E, et al. Effect of everolimus on survival in advanced hepatocellular carcinoma after failure of sorafenib: the EVOLVE-1 randomized clinical trial. JAMA 2014;312:57-67.

76. Zhu AX, Park JO, Ryoo BY, et al. Ramucirumab versus placebo as second-line treatment in patients with advanced hepatocellular carcinoma following first-line therapy with sorafenib (REACH): a randomised, double-blind, multicentre, phase 3 trial. Lancet Oncol 2015;16:859-70.

77. Guichard C, Amaddeo G, Imbeaud S, et al. Integrated analysis of somatic mutations and focal copy-number changes identifies key genes and pathways in hepatocellular carcinoma. Nat Genet 2012;44:694-8.
78. Cleary SP, Jeck WR, Zhao X, et al. Identification of driver genes in hepatocellular carcinoma by exome sequencing. Hepatology 2013;58:1693-702.

79. Semela D, Piguet AC, Kolev M, et al. Vascular remodeling and antitumoral effects of mTOR inhibition in a rat model of hepatocellular carcinoma. J Hepatol 2007;46:840-8.

80. Santoro A, Rimassa L, Borbath I, et al. Tivantinib for second-line treatment of advanced hepatocellular carcinoma: a randomised, placebo-controlled phase 2 study. Lancet Oncol 2013;14:55-63.

81. Cheng AL, Amarapurkar D, Chao Y, et al. Re-evaluating transarterial chemoembolization for the treatment of hepatocellular carcinoma: Consensus recommendations and review by an International Expert Panel. Liver Int 2014;34:174-83.

82. Sergio A, Cristofori C, Cardin R, et al. Transcatheter arterial chemoembolization (TACE) in hepatocellular carcinoma (HCC): the role of angiogenesis and invasiveness. Am J Gastroenterol 2008;103:914-21.

83. Kudo M, Imanaka K, Chida N, et al. Phase III study of sorafenib after transarterial chemoembolisation in Japanese and Korean patients with unresectable hepatocellular carcinoma. Eur J Cancer 2011;47:2117-27.

84. Pawlik TM, Reyes DK, Cosgrove D, et al. Phase II trial of sorafenib combined with concurrent transarterial chemoembolization with drug-eluting beads for hepatocellular carcinoma. J Clin Oncol 2011;29:3960-7.

85. Park JW, Koh YH, Kim HB, et al. Phase II study of concurrent transarterial chemoembolization and sorafenib in patients with unresectable hepatocellular carcinoma. J Hepatol 2012;56:1336-42.

86. Chao Y, Chung YH, Han G, et al. The combination of transcatheter arterial chemoembolization and sorafenib is well tolerated and effective in Asian patients with hepatocellular carcinoma: final results of the START trial. Int J Cancer 2015;136:1458-67.

87. Lencioni R, Llovet JM, Han G, et al. Sorafenib or placebo plus TACE with doxorubicin-eluting beads for intermediate stage HCC: The SPACE trial. J Hepatol 2016;64:1090-8.

88. Geschwind JF, Chapiro J. Sorafenib in combination with transarterial chemoembolization for the treatment of hepatocellular carcinoma. Clin Adv Hematol Oncol 2016;14:585-7.

89. Geschwind JF, Kudo M, Marrero JA, et al. TACE Treatment in Patients with Sorafenib-treated Unresectable Hepatocellular Carcinoma in Clinical Practice: Final Analysis of GIDEON. Radiology 2016;279:630-40. 
90. Larkin J, Chiarion-Sileni V, Gonzalez R, et al. Combined Nivolumab and Ipilimumab or Monotherapy in Untreated Melanoma. N Engl J Med 2015;373:23-34.

91. Borghaei H, Paz-Ares L, Horn L, et al. Nivolumab versus Docetaxel in Advanced Nonsquamous Non-Small-Cell Lung Cancer. N Engl J Med 2015;373:1627-39.

92. Reck M, Rodriguez-Abreu D, Robinson AG, et al. Pembrolizumab versus Chemotherapy for PD-L1Positive Non-Small-Cell Lung Cancer. N Engl J Med 2016;375:1823-33.

93. El-Khoueiry A. Phase I/II safety and antitumo activity of nivolumab (nivo) in patients (pts) with advanced hepatocllular carcinoma (HCC): Interim analysis of the CheckMate-040 dose escalation study 2016. Available online: http://meetinglibrary.asco.org/content/167136-176

94. A Study of Nivolumab Compared to Sorafenib as a Primary
Treatment in Patients with Advanced Hepatocellular Carcinoma [Internet]. 2015 [cited 11/03/2016]. Available online: https://clinicaltrials.gov/ct2/show/NCT02576509

95. Study of Pembrolizumab (MK-3475) vs Best Supportive Care in Participants with Previosuly Treated Advanced Hepatocellular Carcinoma (MK-3475240/KEYNOTE-240) [Internet]. National Library of Medicine. 2016 [cited 03/11/2016]. Available online: https://clinicaltrials.gov/ct2/show/NCT02702401?term =pembrolizumab+AND+Hepatocellular+carcinoma\&ra $\mathrm{nk}=4$

96. Miyayama S, Matsui O, Taki K, et al. Extrahepatic blood supply to hepatocellular carcinoma: angiographic demonstration and transcatheter arterial chemoembolization. Cardiovasc Intervent Radiol 2006;29:39-48.

Cite this article as: Gbolahan OB, Schacht MA, Beckley EW, LaRoche TP, O’Neil BH, Pyko M. Locoregional and systemic therapy for hepatocellular carcinoma. J Gastrointest Oncol 2017;8(2):215-228. doi: 10.21037/jgo.2017.03.13 